\title{
Clinical evaluation of automated capillary refill time estimation in dogs and cats
}

Blaž Cugmas, Eva Štruc, Janis Spigulis

Blaž Cugmas, Eva Štruc, Janis Spigulis, "Clinical evaluation of automated capillary refill time estimation in dogs and cats," Proc. SPIE 10868, Advanced Biomedical and Clinical Diagnostic and Surgical Guidance Systems XVII, 108681N (26 February 2019); doi: 10.1117/12.2507620

SPIE. Event: SPIE BiOS, 2019, San Francisco, California, United States 


\title{
Clinical evaluation of automated capillary refill time estimation in dogs and cats
}

\author{
Blaž Cugmas ${ }^{\mathrm{a} *}$, Eva Štruc ${ }^{\mathrm{b}, \mathrm{c}}$, Jānis Spīgulis ${ }^{\mathrm{a}}$ \\ aBiophotonics Laboratory, Institute of Atomic Physics and Spectroscopy, University of Latvia, \\ Raina Blvd. 19, LV-1586, Riga, Latvia \\ ${ }^{\mathrm{b}}$ Animal Health Centre (Dzīvnieku veselības centrs), F. Candera Str. 4, LV-1046, Riga, Latvia \\ ${ }^{c}$ Vetamplify, veterinary services LLC, K. Valdemara 57/59-32, LV-1010, Riga, Latvia
}

\begin{abstract}
In this study, we clinically evaluated a pulse oximeter-based device for automated capillary refill time (CRT) estimation in dogs and cats. CRT can reveal conditions like shock or anemia in dogs and cats. However, visual CRT estimation has low repeatability, and the available optical systems for automated estimation are not suitable for pets. We evaluated a custom-made portable CRT measuring device on various measurement sites of 12 dogs and 11 cats with parallel visual CRT estimation on the gum by treating veterinarian. The capillary refill was also recorded by a video camera for reference. The visual and video procedures were moderately correlated with the coefficient of 0.61 ; visual CRT values were on average for $0.18 \mathrm{~s}$ longer than the reference. On average, $\sim 32 \%$ of measurements with the proposed device were successful. The rest failed due to excessive pigmentation, motion artifacts, and other pressure-induced effects. The measurement sites of the metacarpal pad, digit, and tail were moderately correlated with the reference values with coefficients of $0.53,0.58$, and 0.42 , respectively.
\end{abstract}

Keywords: Capillary refill time, capillary refill test, dogs, cats, microcirculation, pulse oximeter, photoplethysmography

\section{INTRODUCTION}

In the previous paper ${ }^{1}$, we presented preliminary results with the optical device for automated (i.e., objective) estimation of capillary refill time (CRT). Estimating CRT is a part of the cardiorespiratory examination in dogs and cats ${ }^{2}$, and is normally performed visually by pressing on labial mucosa or the gum above the canine tooth. CRT is the time, which is needed for the normal tissue color to return after the pressure is released. Expected canine and feline CRT is between one and two seconds ${ }^{2}$. Prolonged CRT reflects poor perfusion or peripheral vasoconstriction what can be caused by cold, shock, various cardiovascular diseases or anemia. On the other hand, shorter CRT happens with the various hyperdynamic conditions which are a consequence of pain, fever, heat stroke, distributive shock, etc.

In human medicine, CRT has exhibited high specificity when related to mortality in children so that it can be used as a "red-flag"3. However, it showed low sensitivity, so measuring CRT in the expected range does not carry much of diagnostic value. It was shown that CRT can be influenced by gender, temperature, measurement site and applied pressure $^{4,5}$. Additionally, the visual estimation of CRT has low repeatability ${ }^{6}$, probably due to unstandardized measuring procedure ${ }^{3}$.

Therefore, automated methods have been proposed ${ }^{5,7-9}$, including our optical device ${ }^{1}$. This device solved some shortcomings of the other systems which are big and needed several seconds of patient stillness what is hard to achieve in daily veterinary practice. The influence of animal hairiness and skin pigmentation was reduced as well. As the device from the previous study exhibited problems with controlling applied pressure, it has been modified with calibrated spring and then tested in the clinic on a larger number of patients.

*blaz.cugmas@lu.lv; phone +371 67228249; http://www.asi.lv/

Advanced Biomedical and Clinical Diagnostic and Surgical Guidance Systems XVII edited by Anita Mahadevan-Jansen, Warren S. Grundfest, Proc. of SPIE Vol. 10868

$108681 \mathrm{~N} \cdot$ - C) 2019 SPIE · CCC code: 1605-7422/19/\$18 · doi: 10.1117/12.2507620 


\section{DEVICE}

The optical device (Fig. 1) is a modified version from the previous study ${ }^{1}$ where the operator had difficulties controlling the pressure application. Therefore, a small spring was added, enabling a constant application of pressure. When the spring was fully pressed, $7.48 \mathrm{~N}$ of force was applied. The rest of the device was the same as in the previous study ${ }^{1}$. Briefly, the optical device was based on a pulse oximeter sensor with three LEDs (MAX30105, $5.6 \mathrm{~mm} \times 3.3 \mathrm{~mm}$, $537 / 660 / 880 \mathrm{~nm}$, Maxim Integrated, USA) integrated on the commercially available breakout board $(2.35 \mathrm{~cm} \times 2.09 \mathrm{~cm}$, SEN-14045, SparkFun Electronics, USA). On the reverse side of the sensor, a force resistor (FSR-402, Interlink Electronics, USA) was placed in the voltage divider circuit together with $3.3 \mathrm{k} \Omega$ resistor in order to detect applied pressure. Additionally, the microcontroller (Pro Micro 5V/16MHz, SparkFun Electronics), data logger (DEV-13712, SparkFun Electronics) and 9V battery were also employed.

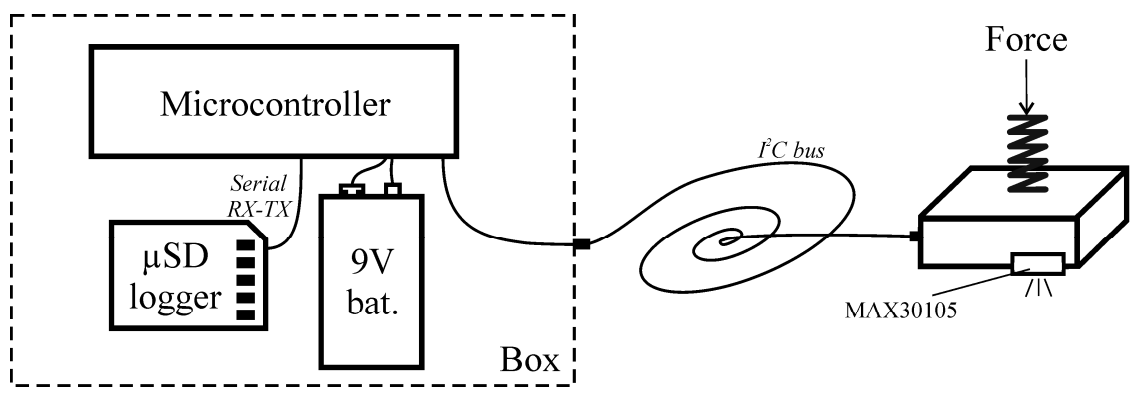

Figure 1. Diagram of the prototype device.

\section{CRT MEASUREMENTS}

The device was tested on 23 pets (12 dogs and 11 cats), which were presented to the clinic for a scheduled dental cleaning. Therefore, the animals were under general anesthesia during the measurements. According to the Latvian national laws and European directive 2010/63/EU, all measurements were led by nationally certified veterinarians, and owner's written permission for the CRT measurement was collected.

Measurements were performed on several measurement sites:

1. labial mucosa (inside the mouth),

2. the gum above canine tooth,

3. the skin above metacarpal bones, between carpal and metacarpal pads (palmar side),

4. metacarpal pad,

5. front leg digit (medial side),

6. the skin above caudal vertebrae (on the tail), close to anal area (ventral side),

7. the chin,

8. the skin above sternum's xiphoid process.

Operator gently leaned the sensor against the tissue and fully compressed the spring for around two seconds, in the same manner as with visual method ${ }^{2}$. Operator ensured that the sensor stayed in good contact with the tissue after the pressure was released. On each measurement site, there were three acquisition repetitions.

As in the previous study ${ }^{1}$, CRT was defined as the difference between optical ( $\left.t_{s 4}\right)$ and pressure $\left(t_{p 3}\right)$ time point (Fig. 2). Briefly, the starting and the ending time points of optical $\left(t_{s 3}, t_{s 4}\right)$ and pressure $\left(t_{p 1}, t_{p 2}, t_{p 3}, t_{p 4}\right)$ signal transitions were calculated with $10 \%$ and $90 \%$ reference levels (risetimelfalltime function, Matlab R2015a, MathWorks Inc, USA). The pressure application duration $\left(t_{p r e s}\right)$ was estimated as a difference between $t_{p 1}$ and $t_{p 3}$. The interval between $t_{p 2}$ and $t_{p 3}$ served for the calculation of the average applied pressure. Finally, CRT was estimated (Fig. 2) as:

$$
C R T=t_{\text {delay }}+t_{\text {fall }}=\left(t_{s 3}-t_{p 3}\right)+\left(t_{s 4}-t_{s 3}\right)=t_{s 4}-t_{p 3},
$$

where $t_{\text {delay }}$ is a delay between the starting points of optical and pressure signal fall and $t_{\text {fall }}$ is a true fall time of the optical signal. 


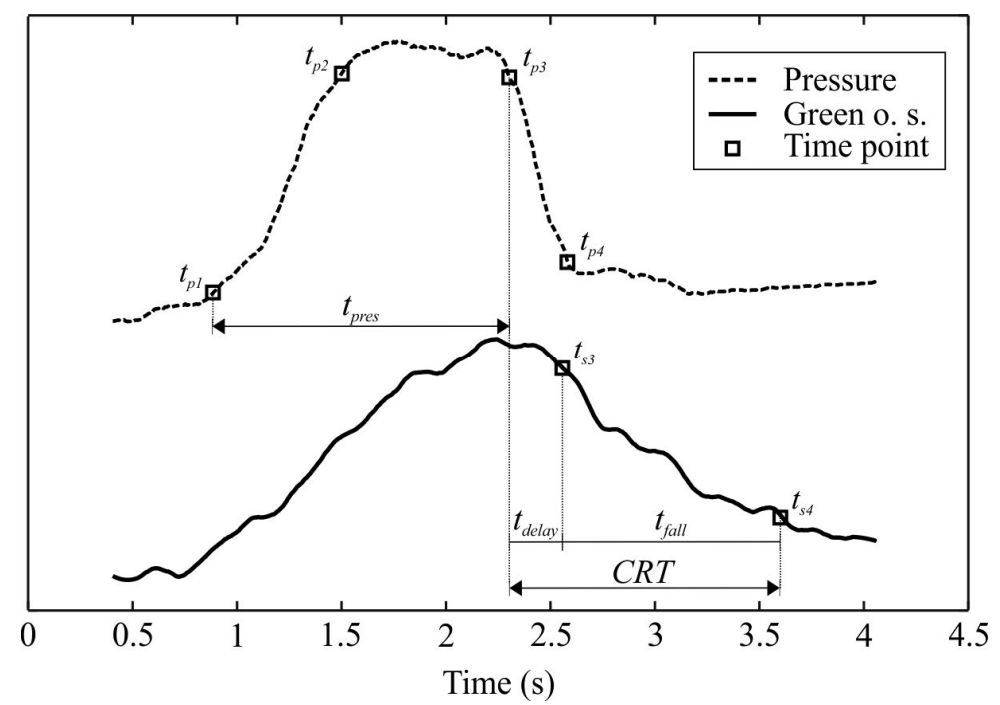

Figure 2 . The starting and ending time points of optical and pressure signal transitions ${ }^{1}$.

In parallel, veterinarian visually estimated CRT by pressing on the gum above the canine tooth. The visual procedure was additionally recorded by the commercial RGB camera ( $\alpha 5100$, Sony, Japan) and by slowly studying the acquired video (25 fps), CRT was estimated as a difference between two events: (1) veterinarian finger stopped pressing and started lifting, (2) the tissue (gum or labial mucosa) returned to the normal red shade. The camera-based CRT estimation was considered as a reference (i.e., the gold standard).

To sum up, CRT was estimated by three methods:

1. by the optical device (Section 2) on several measurement sites,

2. visually by the treating veterinarian (on the gum above the canine tooth),

3. from the video, filming the second method (i.e., the gold standard).

The device successfulness rate was estimated as a ratio between successfully-realized and all measurements. Failed measurements were determined manually by inspecting and comparing optical and pressure signals. Secondly, absolute CRT times were compared between the methods and measurement sites. Finally, the relationship between methods was studied by correlation analysis (Pearson coefficient, corr function, Matlab). Additionally, linear regression was performed with CRT values estimated by the third method (i.e., video) which served as an independent variable (fitlm function, Matlab).

\section{RESULTS AND DISCUSSION}

The successfulness rates of the proposed device at specific measurement sites are listed in Table 1. The average successfulness rate was $31.6 \%$. The labial mucosa, gum, and tail performed better since around $45 \%$ of measurements were successfully realized. When inspecting the acquired signals, the failed measurements can be contributed to three different phenomena (Figure 3). First, skin pigmentation or hairiness heavily absorbed the emitted light what resulted in a weak reflected optical signal. The phenomenon was especially evident on pads. Secondly, motion artifacts appeared. When veterinarian pressed on the spring, optical sensor slid aside changing the optical signal baseline. This predominantly happened on the measurement sites with the firm base (e.g., bone) as the gum above the canine tooth. Finally, the optical signal strictly followed the pressure changes. As speculated in our previous study ${ }^{10}$, these changes are related to tissue hemodynamics which affect absorption and scattering. However, if mainly capillaries are impacted by the pressure induced changes, there should be a slight delay in the optical signal after the pressure was released, but this was not noticed in the failed measurements. 
Table 1. The device successfulness rates (in \%) on different measurement sites. In the brackets, the numbers of successfully-realized and all acquired measurements.

\begin{tabular}{l|ccc}
\hline Site & All (\%) & Dogs $(\%)$ & Cats (\%) \\
\hline 1. Labial mucosa & $45.6(26 / 57)$ & $48.1(13 / 27)$ & $43.3(13 / 30)$ \\
2. Gum & $41.9(18 / 43)$ & $45.8(11 / 24)$ & $36.8(7 / 19)$ \\
3. Metacarpus & $16.9(11 / 65)$ & $16.7(6 / 36)$ & $17.2(5 / 29)$ \\
4. Metacarpal pad & $16.7(6 / 36)$ & $33.3(3 / 9)$ & $11.1(3 / 27)$ \\
5. Digit & $13.5(5 / 37)$ & $12.5(3 / 24)$ & $15.4(2 / 13)$ \\
6. Tail & $46.8(22 / 47)$ & $62.5(15 / 24)$ & $30.4(7 / 23)$ \\
7. Chin & $35.7(10 / 28)$ & $50.0(8 / 16)$ & $16.7(2 / 12)$ \\
8. Sternum & $34.3(12 / 35)$ & $27.8(5 / 18)$ & $41.2(7 / 17)$ \\
\hline Sum & $31.6(110 / 348)$ & $36.0(64 / 178)$ & $27.1(46 / 170)$ \\
\hline
\end{tabular}
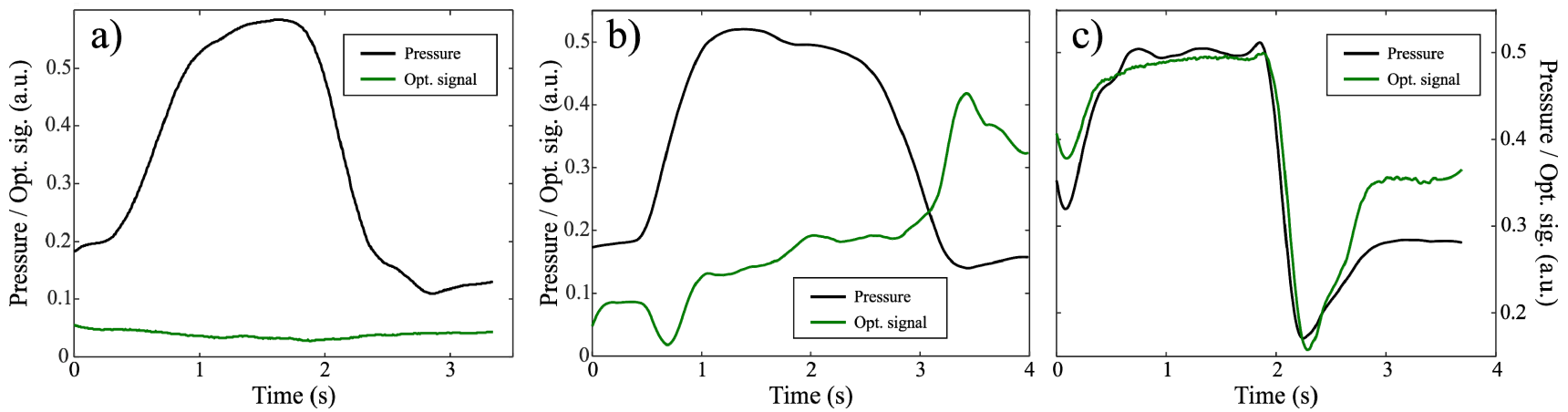

Figure 3. Examples of failed CRT measurements. The black line represents a pressure signal; a green optical signal is denoted by the green line. a) Heavy absorption, metacarpal pad, dog. b) Motion artifact, tail, cat. c) Contact-pressure induced changes, digit, cat.

Table 2. Mean and STD values estimated by three methods: video (V), visually (Vs) and device (D).

\begin{tabular}{l|cc|cccc}
\hline & All & & Dogs & Cats & \\
Method & Mean & STD & Mean & STD & Mean & STD \\
\hline Video (V) & 1.02 & 0.36 & 0.99 & 0.41 & 1.05 & 0.33 \\
Visual (Vs) & 1.20 & 0.57 & 1.02 & 0.51 & 1.40 & 0.59 \\
Difference (Vs-V) & 0.18 & 0.45 & 0.03 & 0.42 & 0.35 & 0.43 \\
\hline All (D) & 0.81 & 0.29 & 0.79 & 0.32 & 0.83 & 0.26 \\
Labial mucosa (D) & 0.86 & 0.27 & 0.79 & 0.20 & 0.93 & 0.33 \\
Gum (D) & 0.84 & 0.42 & 0.93 & 0.52 & 0.71 & 0.21 \\
Metacarpus (D) & 0.65 & 0.09 & 0.64 & 0.10 & 0.67 & 0.08 \\
Metacarpal pad (D) & 0.68 & 0.17 & 0.77 & 0.21 & 0.59 & 0.06 \\
Digit (D) & 0.73 & 0.13 & 0.67 & 0.10 & 0.82 & 0.13 \\
Tail (D) & 0.84 & 0.32 & 0.88 & 0.38 & 0.76 & 0.12 \\
Chin (D) & 0.71 & 0.16 & 0.74 & 0.17 & 0.61 & 0.00 \\
Sternum (D) & 0.76 & 0.17 & 0.67 & 0.21 & 0.83 & 0.12 \\
\hline
\end{tabular}

In Table 2, the mean CRT values with standard deviation (STD) for each measuring method are listed. The mean CRT, estimated from the video, was $1.02 \mathrm{~s}$. The values were ranging from 0.55 to $1.87 \mathrm{~s}$ what is lower than expected values which should be between one and two seconds ${ }^{2}$. The video measurements were done on the gum of dental patients, where their infected gums often exhibited hyperemia, hyperdynamic conditions, which decreased CRT. Visually, 
estimated CRT was on average for $0.18 \mathrm{~s}$ longer. The feline patients predominantly contributed to this difference. One reason for the difference can be found in the lower color contrast between the normal and compressed gum in some cats. Consequently, it was harder to visually detect the returned tissue color. CRT values, estimated with the proposed device, were in general lower, ranging from 0.65 (metacarpus) to $0.86 \mathrm{~s}$ (labial mucosa).

Since estimating CRT on the gum or labial mucosa is widely considered as a gold standard in the veterinary practice, both, visual and device-based methods were compared with the video estimation by correlation and linear regression methods. The results are collected in Table 3. The video and visual methods were related the most since there was a moderate correlation (Figure 4(a)). The slope of the fitted line $(a)$ was almost one $(a=0.96)$; the additive constant $(b)$ was $0.22 \mathrm{~s}$, close to the mean method difference (Table 2). Despite the gum and labial mucosa exhibited high successfulness rate, the correlation coefficient was extremely low. Therefore, we cannot be sure if CRT was measured. Probably, some other phenomenon influenced the estimated data.

On the other hand, metacarpal pad and digit exhibited moderate correlation, similar to the visual approach. This is not surprising since CRT in humans is most often estimated on fingers. However, both measurement sites exhibited low successfulness rates, meaning in most of the animals (around 83\%) measurements were not successful. The tail can be an alternative since it exhibited some correlation and satisfying successfulness rate (Figure 4(b)).

Table 3. The correlation coefficient between a method and video (V) CRT estimation. $\mathrm{R}^{2}$ and fitted function from linear regression are additionally listed.

\begin{tabular}{l|ccc}
\hline \multirow{2}{*}{ Method } & $\begin{array}{c}\text { Correlation } \\
\text { coefficient }\end{array}$ & $\mathrm{R}^{2}$ & $\begin{array}{c}\text { Linear regression } \\
\text { Fitted function } \\
(a \mathrm{~V}+b)\end{array}$ \\
\hline Visual & 0.61 & 0.38 & $0.96 \mathrm{~V}+0.22$ \\
Labial mucosa (D) & 0.14 & 0.02 & $0.74 \mathrm{~V}+0.13$ \\
Gum (D) & -0.24 & 0.06 & $1.23 \mathrm{~V}-0.47$ \\
Metacarpus (D) & 0.27 & 0.07 & $0.48 \mathrm{~V}+0.26$ \\
Metacarpal pad (D) & 0.53 & 0.28 & $0.40 \mathrm{~V}+0.31$ \\
Digit (D) & 0.58 & 0.33 & $0.57 \mathrm{~V}+0.14$ \\
Tail (D) & 0.42 & 0.18 & $0.48 \mathrm{~V}+0.36$ \\
Chin (D) & -0.41 & 0.17 & $0.84 \mathrm{~V}-0.15$ \\
Sternum (D) & 0.00 & 0.00 & $0.73 \mathrm{~V}+0.00$ \\
\hline
\end{tabular}
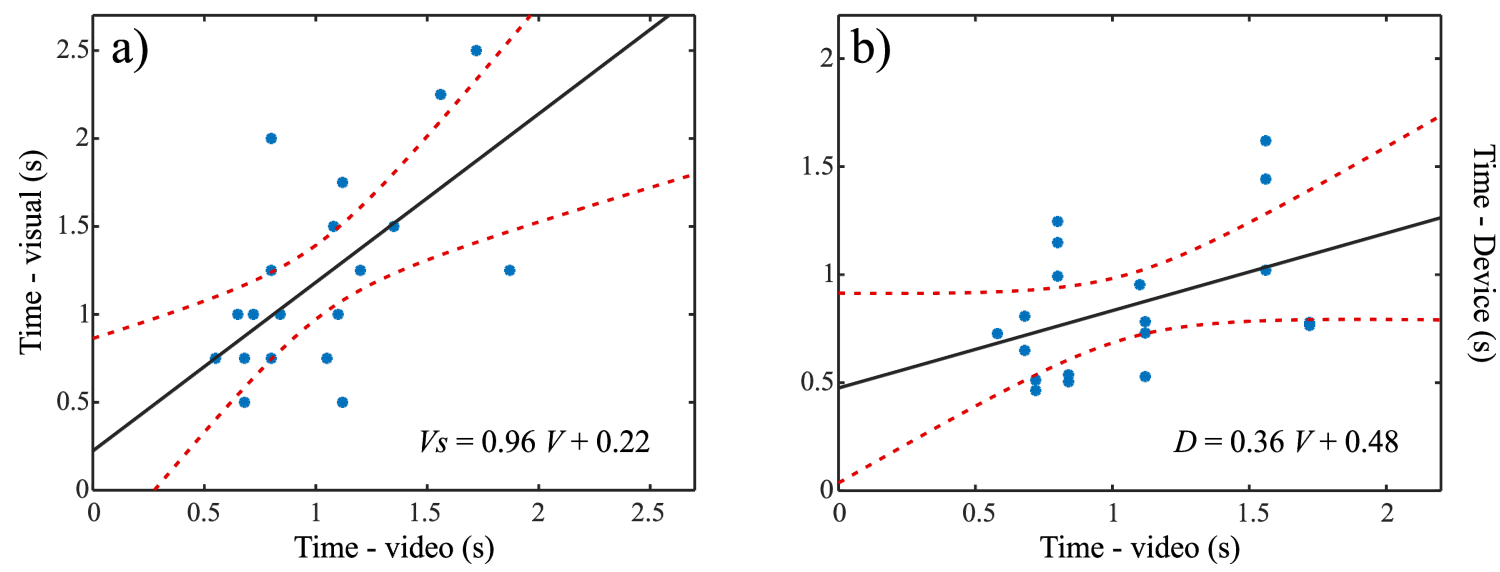

Figure 4. CRT estimated a) by video $(V)$ and visual method $(V s)$, and b) by video $(V)$ and Device method (the tail) $(D)$. The fitted regression line is plotted with $95 \%$ confidence intervals. 


\section{CONCLUSION}

Despite all the efforts, automated CRT estimation is still not successfully applied to the clinics. John et al. recently added that further investigation of protocols, interpretation algorithms and guidelines is needed ${ }^{5}$. As our study with pets revealed, a more accurate and robust device will be needed in veterinary practice since the proposed device was reliable only on the metacarpal pad, digit, and tail. Moreover, many measurements were affected by heavy pigmentation, motion artifacts, and other contact-pressure related effects. Possibly, the camera-based approach could work well on nonpigmented and easy-accessible measurement sites.

\section{ACKNOWLEDGMENTS}

The study was supported by the Marie Skłodowska-Curie actions of the European Union (IF, DogSPEC, 745396). We would also like to express our gratitude to veterinarians Aigars Brinkisis, Liene Dindone, Alla Olivrī, Solvita Buivide and all other personnel from Animal Health Centre and Vetamplify for all the help and technical support.

\section{REFERENCES}

[1] Cugmas, B. and Spigulis, J., "Challenges in automated estimation of capillary refill time in dogs," Proc. SPIE 10501, 1050117 (2018).

[2] Bexfield, N. and Lee, K., [BSAVA Guide to Procedures in Small Animal Practice], British Small Animal Veterinary Association, Gloucester (2010).

[3] Fleming, S., Gill, P., Jones, C., Taylor, J. A., Van den Bruel, A., Heneghan, C., Roberts, N. and Thompson, M., "The Diagnostic Value of Capillary Refill Time for Detecting Serious Illness in Children: A Systematic Review and Meta-Analysis," PLoS ONE 10(9), e0138155 (2015).

[4] Pickard, A., Karlen, W. and Ansermino, J. M., "Capillary Refill Time: Is It Still a Useful Clinical Sign?” Anesth. Analg. 113(1), 120-123 (2011).

[5] John, R. T., Henricson, J., Nilsson, G. E., Wilhelms, D. and Anderson, C. D., "Reflectance spectroscopy: to shed new light on the capillary refill test," J. Biophotonics 11(1), 1-9 (2017).

[6] Pickard, A., Stinson, J., Cooke, E., Myers, D., Karlen, W. and Ansermino, J. M., "Intra-observer and interobserver repeatability of capillary refill time," Anaesthesia 67, 44-44 (2012).

[7] Faezeh Talebi Liasi, Ravikant Samatham and Steven L. Jacques., "Noninvasive in vivo optical characterization of blood flow and oxygen consumption in the superficial plexus of skin," J. Biomed. Opt. 22(11), 115002 (2017).

[8] Shamsudin, N., "A Device for Measurement of Capillary Refilling Time," Master's thesis, Linköping University, Linköping (2012).

[9] Blaxter, L. L., Morris, D. E., Crowe, J. A., Henry, C., Hill, S., Sharkey, D., Vyas, H. and Hayes-Gill, B. R., “An automated quasi-continuous capillary refill timing device," Physiol. Meas. 37(1), 83 (2016).

[10] Cugmas, B., Bregar, M., Bürmen, M., Pernuš, F. and Likar, B., "Impact of contact pressure-induced spectral changes on soft-tissue classification in diffuse reflectance spectroscopy: problems and solutions," J. Biomed. Opt. 19(3), 037002 (2014). 